\title{
Pheasants Learn Five Different Binomial Color Discriminations and Retain these Associations for at Least 27 Days
}

\author{
Jayden O. van Horik*, Ellis J.G. Langley, Mark A. Whiteside, \\ Christine E. Beardsworth and Joah R. Madden
}

Centre for Research in Animal Behavior, Psychology, University of Exeter, UK

*Corresponding author (Email: jayden.van.horik@gmail.com)

Citation - van Horik, J. O., Langley, E. J. G., Whiteside, M. A., Beardsworth, C. E., \& Madden, J. R. (2018). Pheasants learn five different binomial color discriminations and retain these associations for at least 27 days. Animal Behavior and Cognition, 5(3), 268-278. https://doi.org/10.26451/abc.05.03.02.2018

\begin{abstract}
Individuals likely vary in how quickly they learn, how many different associations they may be able to maintain, and how long they can remember previously learned associations. However, it is unclear whether capacities for these cognitive processes are consistent within individuals, or whether individual performance differs when presented with novel variants of tasks, or across tasks that assess different cognitive abilities. We investigate associative learning and long-term reference memory in young (3-8 week-old) pheasants (Phasianus colchicus) using a series of visual binary discrimination problems that were presented at different intervals of time. Birds were sequentially presented with five novel color pairs, where they could learn that one color of each pair was consistently rewarded. After experiencing these learning trials, subjects were re-tested on each discrimination problem, but at different intervals (0-27 days), to assess their memory. Subjects' learning performance improved within 50 trials of each discrimination problem. We found no differences in performance between final learning sessions and initial memory sessions across the different time intervals, suggesting that pheasants retained the previously learned associations of multiple color pairs over at least a 27-day interval. Moreover, proactive interference did not impede their ability to recall subsequently learned color contingencies. Although individual learning and memory performance correlated positively, we found no evidence that individuals' performances were consistent across task variants. Our findings illuminate capacities for associative learning and long-term reference memory in pheasants.
\end{abstract}

Keywords - Color discriminations, Cognition, Learning, Memory, Phasianus colchicus

Animals encounter many different stimuli in their environment and, through experience, may benefit from learning the affordances of these interactions. Two cognitive processes, learning and memory, likely play important roles in underpinning these interactions (Healy \& Jones, 2002; Shettleworth, 2010). Memory has been defined as a behavioral change caused by an experience, and learning as a process for acquiring memory (Okano, Hirano, \& Balaban, 2000). Learning and remembering visual discriminations has been found to aid foraging success (Raine \& Chittka, 2008) and may be particularly useful in heterogeneous environments (Boyer \& Walsh, 2010) or when food availability fluctuates seasonally (Roth, LaDage, Freas, \& Pravosudov, 2012; Roth \& Pravosudov, 2009). Long-term memory may also aid navigation, such as required by migratory species (Lohmann, Luschi, \& Hays, 2008; Mettke-Hofmann \& Gwinner, 2003). An ability to learn and remember discriminations may also improve social interactions, for example when distinguishing familiar from unfamiliar individuals or 
attending to social hierarchies (Bird \& Emery, 2008; Boeckle \& Bugnyar, 2012; Bradshaw, 1991; Wascher, Szipl, Boeckle, \& Wilkinson, 2012).

Whereas learning and memory may aid an individual's interactions with their environment, capacities for these processes may also be constrained by cognitive costs. For example, the ability to learn and recall multiple discriminations can become impaired by subsequently learned associations. This may result from retrieval competition from previously learned associations through processes of proactive interference (Keppel \& Underwood, 1962). Hence, when presented with a series of discrimination problems, it may be more difficult to recall recently learnt discriminations than those learnt previously. Serial position effects (primacy and recency) may also facilitate the retention of early or later learnt cues, whereas those presented in-between suffer poor recollection (Gaffan, 1992). Whereas primacy effects have been traditionally considered associated with long-term memory and recency effects with short-term memory, humans, primates and birds all show these serial position effects; suggesting that they share similar mechanisms of memory systems (Sands \& Wright, 1980; Santiago \& Wright, 1984; Wright, Santiago, Sands, Kendrick, \& Cook, 1985). Memories may also decay over time, and as such, initially learnt discriminations may be more difficult to recall than those that were more recently learnt (Brown, Neath, \& Chater, 2007). Consequently, there may be a number of different cognitive processes that constrain an individual's ability to retain and recall previously learned information.

There is a vast literature on the different kinds of learning and memory in humans and animals. In humans, 256 different kinds of memory have been collated (Tulving, 2007), with broad distinctions categorized under: explicit and implicit, conscious and unconscious, voluntary and involuntary retention, intentional and incidental learning and retrieval, declarative and procedural, and retrospective and prospective (Roediger, Zaromb, \& Goode, 2009). Animals also use a variety of different types of memory (see Shettleworth, 2010). Some examples include long-term, spatial and episodic-like memory used by caching birds (Balda \& Kamil, 1992; Clayton \& Dickinson, 1998; van Horik \& Burns, 2007), working spatial memory in rats (Dudchenko, 2004; Olton, Collison, \& Werz, 1977), associative visual, working and reference memory in pigeons (Cook, Levison, Gillett, \& Blaisdell, 2005; Roberts, Strang, \& Macpherson, 2015; Vaughan \& Greene, 1984), long-term and so-called anesthesia-resistant memory in Drosophila (Mery \& Kawecki, 2005), and prospective memory in apes and corvids (Mulcahy \& Call, 2006; Osvath, 2009; Raby, Alexis, Dickinson, \& Clayton, 2007; Raby \& Clayton, 2009), among many others. Likewise, different processes govern learning in animals, such as habituation, imprinting, Pavlovian (classical), operant and associative learning (Churchland, 1986; Mackintosh, 1974; Rescorla, 1988; Shettleworth, 2010). However, the criteria by which the varieties of learning and memory have been distinguished remain unclear and have resulted in a classification problem (Shettleworth, 1993).

Despite the apparent importance of learning and memory, little is known about these processes using non-invasive techniques in Galliformes, which are widely used for studies of cognition (Marino, 2017). Moreover, it remains unclear whether inter-individual variation in learning and memory performance are consistent across similar tasks, or whether an individual's learning performance is related to their memory performance. Pheasants (Phasianus colchicus) provide a unique opportunity to address these questions as they can be reared and tested in large numbers under standardized conditions. Little is currently known about learning, memory, and color vision in pheasants. We therefore assess individual learning and memory performance of multiple color discrimination tasks, across different time intervals, and in an environment in which we standardized individual variation in experience. To investigate learning performance, we presented individual pheasants with a series of binary discrimination problems involving five novel color pairs. For each color pair, one color was consistently rewarded while the other color remained unrewarded. Birds could therefore learn to differentiate between the two choices by associating rewarded and unrewarded options with particular color cues. Hence, we consider that associative learning processes underlie their performance on this visual discrimination task. After experiencing each of the five binary discrimination tasks, subjects were re-tested on the previously learned color contingencies of each color pair at intervals ranging between zero and 27 days. We consider our tasks to assess reference memory, as the ability to recall each previously learned color contingency involves memories formed for the unchanging characteristics of a task, i.e., that one color is rewarded 
while the other is not (Honig, 1978; Roberts et al., 2015). Moreover, we also consider that the ability to recall contingencies involves processes of long-term visual memory (Cook et al., 2005), as color pairs were presented at intervals of up to 27 days. If pheasants show capacities to retain previously learnt information, we expect their performance on initial memory sessions not to differ from those of the final learning sessions. If previously learned discriminations interfere with their ability to recall subsequently learned discriminations, we expect relatively poor performance on memory sessions during latter learnt discriminations compared to initially learnt discriminations. We also assess the consistency of interindividual variation in learning and memory performance and whether an individual's learning performance relates to their memory performance.

\section{Subjects}

Method

One hundred pheasant chicks were hatched in incubators, housed in groups of 50 in two replicated enclosures and reared from one day old between 17 May 2016 and 22 July 2016. All subjects were individually marked using numbered wing tags, fed on commercial pheasant feed (Keepers' Choice) and supplied with water ad libertum. Birds were housed in $2 \mathrm{~m} \times 2 \mathrm{~m}$ heated pens for the first two weeks of life. They had access to unheated, but covered outdoor runs of $1 \mathrm{~m} \mathrm{x} 4 \mathrm{~m}$ for the next week. For the final seven weeks of rearing, each group had additional access to separate $4 \mathrm{~m} \mathrm{x} 12 \mathrm{~m}$ outdoor enclosures. Prior to testing, birds were habituated to the experimental chamber and shaped to peck though crepe paper into wells that would be subsequently used to assess their learning and memory performance (see van Horik, Langley, Whiteside, \& Madden, 2017). All birds were tested from 17-days-old, with equal exposure in a fixed order to all tasks. Birds were individually tested in experimental chambers $(75 \mathrm{~cm} \mathrm{x}$ $75 \mathrm{~cm}$ ) which, during testing times, they could enter at will and where they were visually isolated from other birds.

All applicable international, national, and/or institutional guidelines for the care and use of animals were followed. All work was conducted under Home Office license PPL 30/3204 and approved by the University of Exeter Animal Welfare Ethical Review Board. Birds were habituated to human observation from one day old. Shaping procedures, using meal-worm rewards, were adopted to habituate subjects to the testing arena. These procedures were considered to mitigate stress and encouraged subjects' voluntarily participation during testing. Birds could therefore choose whether to participate in tasks. There were no enforced aversive stimuli. Birds that failed to engage with the task in $<2$ min were permitted to pass into the recovery area and their lack of participation recorded. Birds were reared at a lower density than that recommended by DEFRA's code of practice (DEFRA, 2009), thus likely reducing stress and competition between chicks.

\section{Testing Procedures}

During testing, subjects were required to discriminate between two wells, each $15 \mathrm{~mm}$ in diameter and positioned $20 \mathrm{~mm}$ apart, in which the contents were concealed by a layer of crepe paper. Scent cues were controlled for by using mealworm extracts on the base of all wells. One well contained a mealworm reward while the other well was made inaccessible by covering it with hard black card placed under the crepe paper, which could not be pecked through. Each well was encircled by a different color cue, with the location of the rewarded well counterbalanced across trials so that it was not presented in the same position (top or bottom) for more than three consecutive trials. A correct choice was scored if subjects first pecked into a rewarded well and an incorrect choice was scored if subjects first pecked at an unrewarded well. If the bird made a correct choice, it was allowed to eat the reward. If the bird made a wrong choice, the pair of wells was removed and replaced with a new pair, constituting a new trial. Birds were tested from Mondays to Fridays and each subject received two ten-trial sessions per day, one in the morning and one in the afternoon. Hence, subjects experienced their first 10 trials (session 1) of a given color pair on a Monday morning and were presented with their final 10 trials (session 5) of that color pair 
on a Wednesday morning. Subjects then experienced their first 10 trials (session 1) of a subsequent color pair on a Wednesday afternoon, and were presented with their final 10 trials (session 5) of that pair on a Friday afternoon. Testing ceased when birds were approximately 10 weeks old. All birds were then transferred into a soft-release enclosure in the wild, where they would be the focus of subsequent studies. This enclosure was surrounded by a predator proof fence to protect birds from mammalian predators but had an open top so that their dispersal into the surrounding environment was not prohibited.

Learning and Memory Discriminations. Birds were presented with five tasks involving novel color pairs. Each color pair comprised a Learning phase and a subsequent Memory phase (Table 1). Subjects were presented with five 10-trial sessions on each color pair during the Learning and Memory phases. All birds received Learning tasks in the same order. After all the Learning tasks were completed, we presented birds with the five Memory tasks. These tasks used the same color pairs as in the Learning tasks, but they were presented in the opposite order. As such, the colors of the last Learning task pair were used in the first Memory task, whereas the colors used in the first Learning pair were used in the last Memory task (Table 1).

Table 1

Presentation Order and Test Dates of Color Learning (L1-L5) and Memory (M1-M5) Discriminations for each Color Pair

\begin{tabular}{lccc}
\hline \multicolumn{1}{c}{ Color Contingency } & Learning & Memory & Interval (days) \\
\hline L1;M5 (Blue+/Light Green-) & 6,7,8 June & $06,07,08$ July & 27 \\
L2;M4 (Yellow+/Pink-) & 8,9,10 June & 04,05,06 July & 23 \\
L3;M3 (Black+/White-) & 13,14,15 June & 29,30 June; 01 July & 13 \\
L4;M2 (Red+/Dark Green-) & 15,16,17 June & $27,28,29$ June & 9 \\
L5;M1 (Orange+/Beige-) & 20,21,22 June & 22,23,24 June & 0 \\
\hline
\end{tabular}

Statistical Analyses. To standardize each individual's experience, we included only birds that had completed all 50 trials on each of the five Learning and Memory discriminations in analyses (i.e., 500 trials: Males $n=39$; Females $n=19$ ). All analyses were conducted in SPSS (IBM Corp, 2013) unless otherwise stated.

(1) Do pheasant chicks show biases towards particular colors?

We used Binomial tests (set at 0.5) to determine whether subjects' first trial choices on each discrimination task differed from chance, and hence whether, at the population level, subjects showed any inherent biases for or against particular colors during the Learning tasks.

(2) Do pheasants improve their performance within 50 trials on each color discrimination task?

To determine whether subjects learned each color association, we compared an individual's number of correct choices, as a measure of their performance, in their first 10-trial session for each color pair with the number of correct choices on their final (fifth) session for that same pair using Repeated Measures ANOVA (RM ANOVA). We included Improvement (in performance) as a factor with two levels: (1) first learning session correct choices and (2) final learning session correct choices. We also included Color Pair as a factor with five levels; one level for each color pair that was presented to subjects. Two-way interactions between Improvement and Color Pairs were included.

(3) Can pheasants retain the previously learned reward contingencies of different color pairs when retested after intervals of up to 27 days?

We used Binomial tests (set at 0.5) to assess whether birds were more likely to make a correct choice on their first trial on the Memory tasks and hence whether subjects, at the population level, remembered each color association across the varying time intervals. We further assessed memory by comparing performances on the final Learning discriminations with those of the first Memory discriminations using RM ANOVA. We included Retention as a factor with two levels: (1) final learning session correct choices and (2) first memory session correct choices (inversed so that each color pair was matched to the re-test interval of each respective pair). We also included Color Pair as a factor with five 
levels; one level for each color pair that was presented to subjects. Two-way interactions between Retention and Color Pairs were included.

(4) Is an individual's learning performance related to their memory performance?

To determine whether individual performance on the Learning discriminations was related to Memory performance, we summed each individual's correct choices across the five sessions for each color pair to provide overall Learning and overall Memory performance measures. We compared individual overall Learning performance with overall Memory performance using Spearman's rank correlations.

We also assessed whether improvement across the learning trials influenced initial memory performance. Improvement was scored as the difference between correct choices made on the final 10 trials of a particular color pair and those made on the first 10 trials of that corresponding color pair. Individuals that showed negative improvement were scored as zero. Memory was scored as the number of correct choices made in the first 10 trials of a given color pair. We used Generalized Linear MixedEffects Models (GLMM), with a Poisson error distribution in R version 1.0.143 (R Development Core Team, 2014) using the lme4 package (Bates, Maechler, Bolker, \& Walker, 2015). We included improvement as our predictor variable and memory as our response variable. We included Bird Identity and Task (each of the five different color pairs) as random effects to control for pseudoreplication.

(5) Is individual learning performance repeatable?

We assessed individual repeatability in performance (see code below) both within the five Learning (Model 1) and five Memory tasks (Model 2), and for cumulative performances (summed correct choices) between Learning and Memory tasks (Model 3) using the rptR package (Stoffel, Nakagawa, \& Schielzeth, 2017), in R (R Development Core Team, 2014) using Generalized Linear Mixed Models (GLMM) with a Poisson error distribution following (Nakagawa \& Schielzeth, 2010).

Code for Repeatability estimates:

Model_1<rpt(Learning $\sim(1 \mid$ Bird $)$,grname=c("Bird"),data=LearnMem,datatype="Poisson")

Model_2<rpt(Memory (1|Bird), grname=c("Bird"),data=LearnMem,datatype="Poisson")

Model_3<rpt(Correct $\sim(1 \mid$ Bird $)$, grname=c("Bird"),data=TotalLearnMem,datatype="Poisson")

\section{Results}

(1) Do pheasant chicks show biases towards particular colors?

At the population level, first trial choices in three of the Learning tasks did not differ from chance (Binomial test; Figure 1). First trial choices in two of the Learning tasks showed biases towards pink (unrewarded) over yellow in their second color pairing (L2) and orange (rewarded) over beige in their fifth color pairing (L5; Binomial test; Figure 1). However, subjects performed at chance on the first 10 trials of the Learning sessions for each color pair (Figure 2), and hence, did not appear to exhibit stable biases for or against colors of any given pair.

(2) Do pheasants improve their performance within 50 trials on each color discrimination task?

Individuals' performance improved between their first and final sessions during the Learning discriminations of five novel color pairs (RM ANOVA: $\mathrm{F}(1,57)=98.87, p<.001$, effect size $=0.634$, Figure 2). Performances differed across the five color pairs (RM ANOVA: F $(4,228)=3.489, p=.009$, effect size $=0.058$, Figure 2). There was a significant interaction between Improvement and Color Pair $($ RM ANOVA: $\mathrm{F}(4,228)=4.411, p=.002$, effect size $=0.072$, Figure 2$)$.

(3) Can pheasants retain the previously learned reward contingencies of different color pairs when retested after intervals of up to 27 days?

Individuals were more likely to choose the rewarded, rather than unrewarded, color on their first choices of the Memory tasks in three of the five color pairs (M1; M3 and M5), suggesting that they retained the associations of these previously learned color contingencies (Binomial test; Figure 1). There was no effect of Retention, suggesting no differences in performance between the final Learning sessions and the first Memory sessions across the different re-test intervals of each color pair (RM ANOVA: $F(1$, $57)=0.910, p=.344$, effect size $=0.016$; Figure 2). Performances differed across the five Color Pairs 
$($ RM ANOVA: $F(4,228)=7.571, p<.001$, effect size $=0.117$, Figure 2$)$. There was no significant interaction between Retention and Color Pair (RM ANOVA: $F(4,228)=1.732, p=.144$, effect size $=$ 0.029, Figure 2).

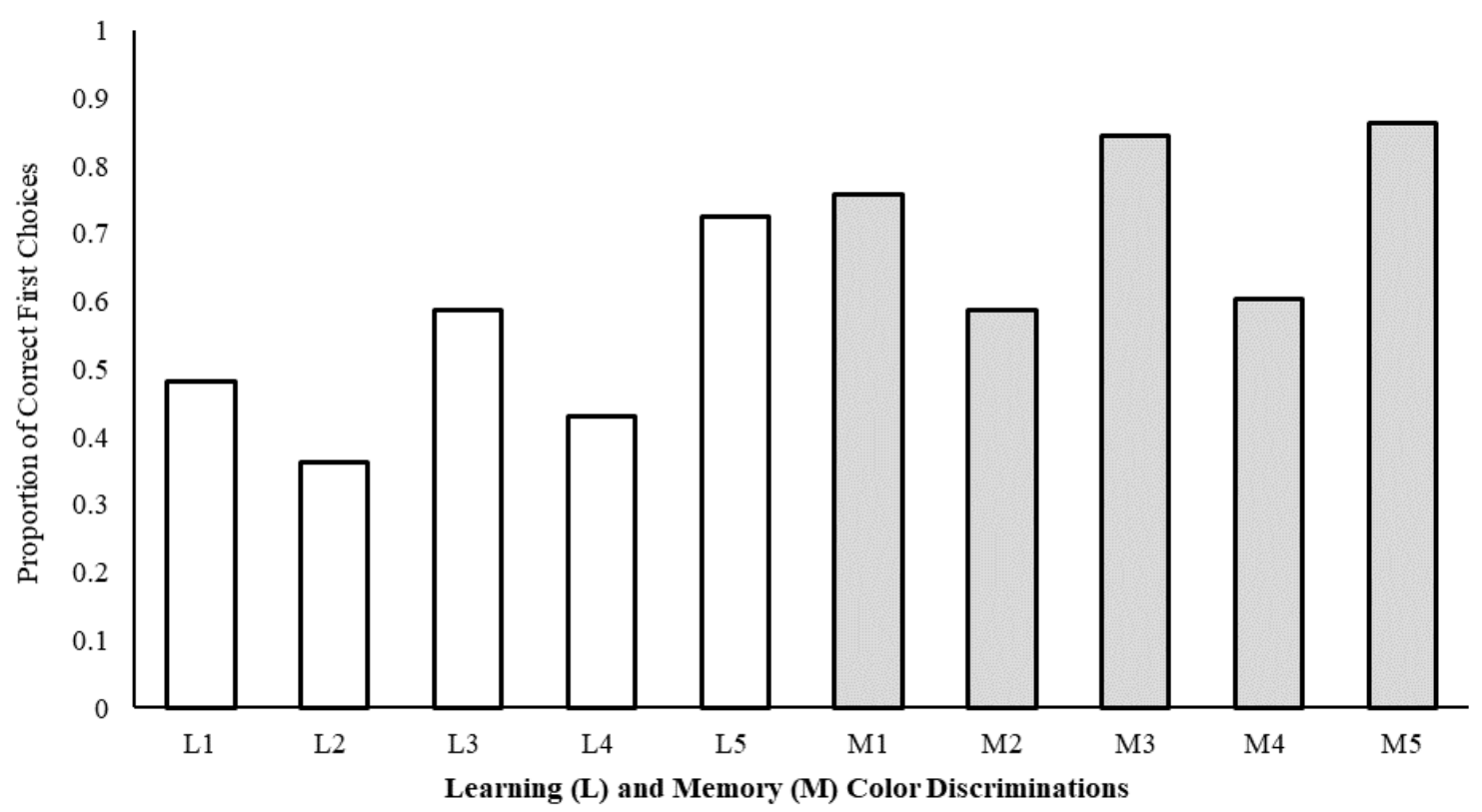

Figure 1. Proportion of correct first trial choices on Learning (white bins L1-L5) and Memory discriminations (grey bins M1M5) of color pairs (presented in chronological order of testing). Binomial test $\mathrm{P}$ values are presented in parentheses. Color contingencies for Learning and Memory tasks are presented in Table 1. The re-test intervals (days) between each respective color pair are presented in Table 1. Significant differences $(p \leq .05)$ between the proportion of correct and incorrect choices as derived from Binomial tests are as follows: L1: $p=0.9$; L2: $p=0.05$; L3: $p=.24$; L4: $p=.36$; L5: $p=.001 ; \mathrm{M} 1: p<.001 ; \mathrm{M} 2: p=.24$; M3 $p<.001 ; \mathrm{M} 4: p=.15 ; \mathrm{M} 5: p<.001$.

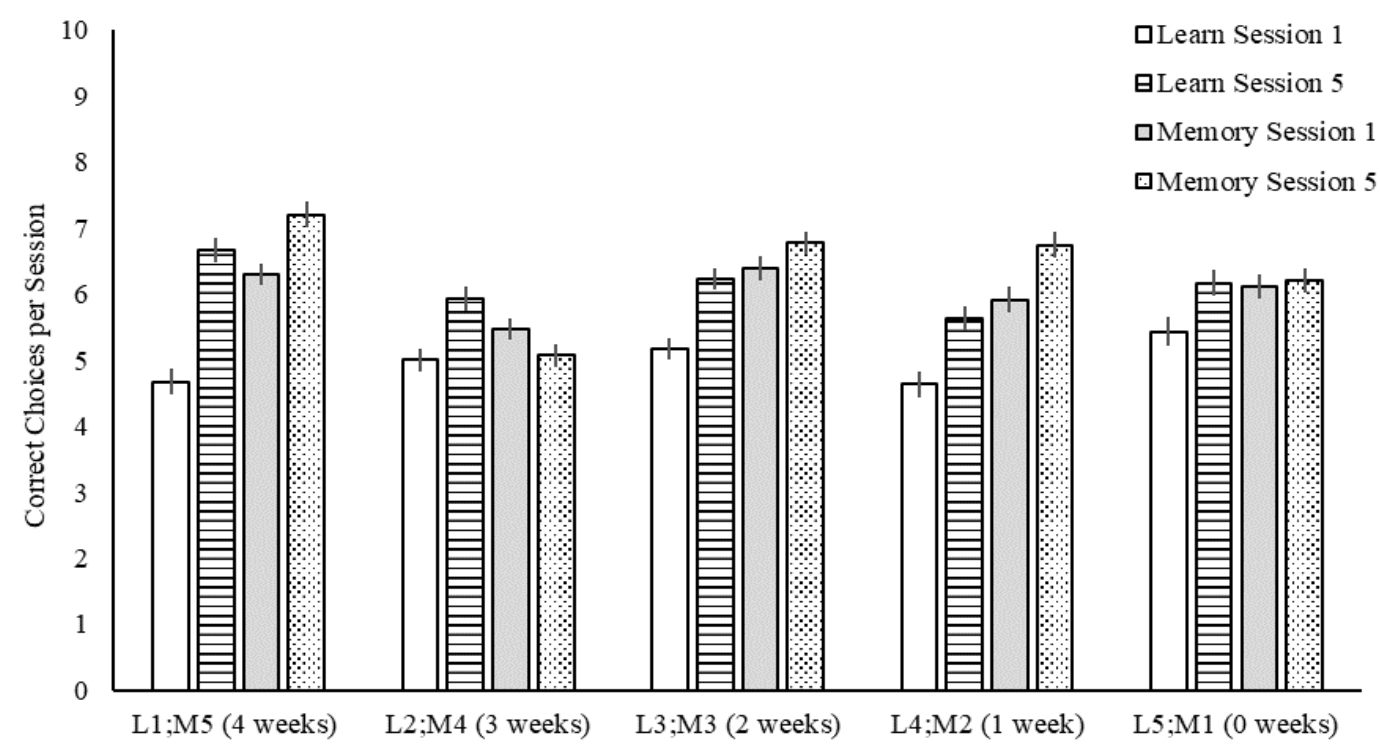

Figure 2. Mean Learning and Memory performance ( \pm SEM) in the first (Session 1) and final (Session 5) 10-trial sessions of five color discrimination tasks. Retest time intervals for Memory trials are presented in parentheses. 
(4) Is an individual's learning performance related to their memory performance?

An individual's overall number of correct choices, summed across all color pairs, correlated positively between summed Learning and Memory tasks (Spearman's Rank Correlation: $R \mathrm{~s}=0.581, p=$ $<.001, n=58$, Figure 3). However, improvement in learning performance was not associated with initial memory performance $(\mathrm{GLMM}): Z=0.38, \pm 0.016 \mathrm{SEM}$, Estimate $=0.006, p=.702$.

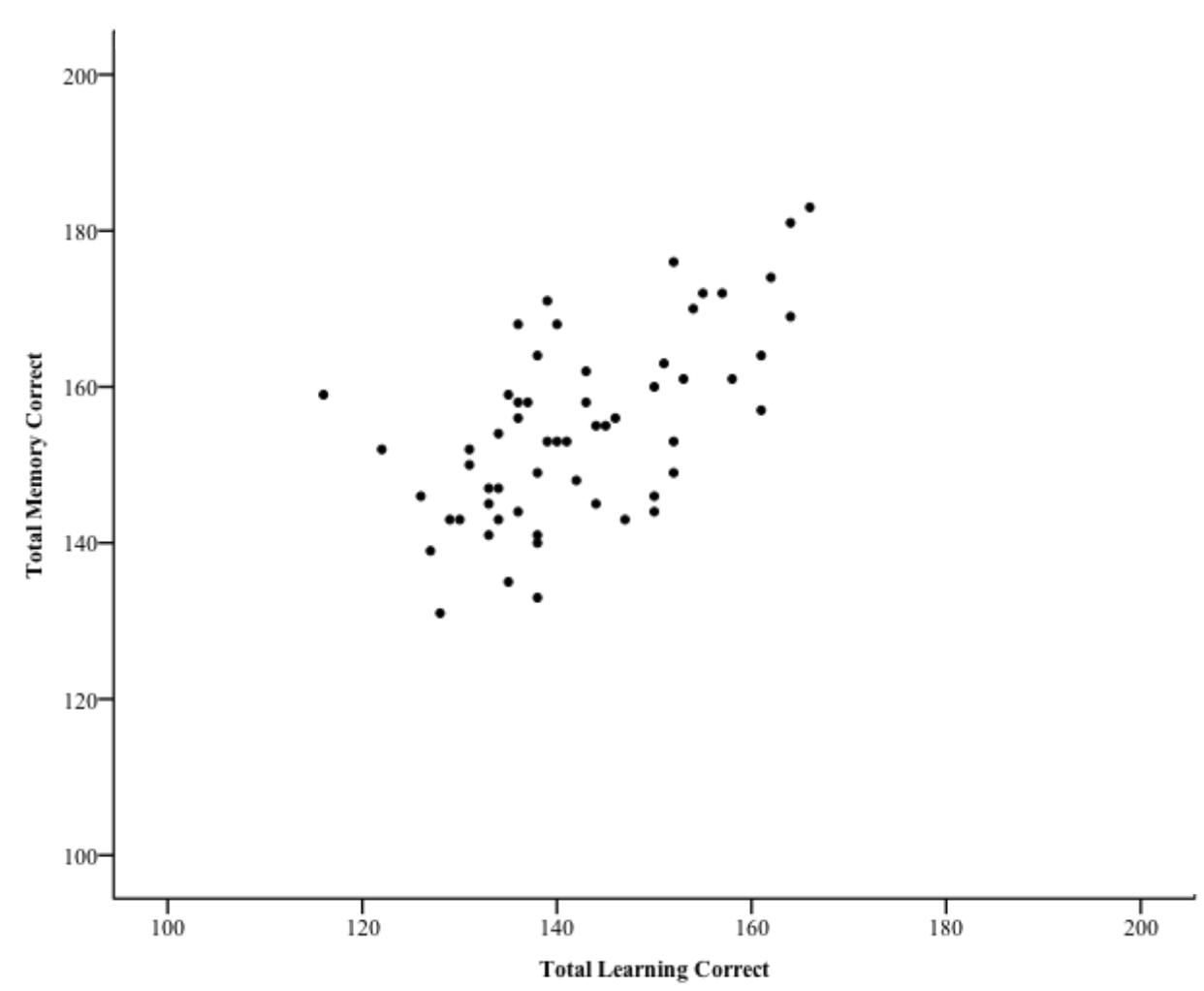

Figure 3. Overall correct choices made during Learning and Memory discriminations.

(5) Is individual learning performance repeatable?

We found no evidence supporting repeatable individual performance, within both the five Learning $(R=0$, CIs [0, 0.064], $p=1)$ and five Memory tasks $(R=0$, CIs [0, 0.068], $p=1)$, or between summed Learning and Memory tasks $(R=0.20$, CIs $[0,0.34], p=.05)$.

\section{Discussion}

At a population level, young pheasants showed an improvement in their ability to discriminate between rewarded and unrewarded colors within 50 trials, across each of five novel color pairs. Birds initially performed at chance in their first 10 trials for each color pair, but improved their performance to approximately $60 \%$ success in their final 10 trials. Pheasants could accurately retain the previously learned reward contingencies of particular color pairs, showing similar performances when re-tested at intervals of up to 27 days. We found no evidence that their performance decreased with an increase in retest intervals between their initial Learning task and the corresponding Memory tasks. These findings suggest that neither subsequent task-specific information (i.e., proactive interference or primacy/recency effects) nor temporal delays degraded the recollection of the reward contingencies of five conceptually similar discriminations. However, although individual performance on the learning tasks correlated positively with performance on the memory tasks, we found no evidence to suggest that individual 
learning and memory performance was repeatable within the five color pairs or across summed learning and memory discriminations.

We show that pheasants can learn the reward contingencies of five different color pairs and that their capacities to retain these associations do not diminish over intervals of up to 27 days. We consider the ability to retain and recall the contingencies of each of the five different color pairs to highlight pheasants capacities for long-term reference memory (Cook et al., 2005; Honig, 1978; Roberts et al., 2015). There are however different kinds of long-term memory (Honig \& James, 1971; Roediger et al., 2009) and it remains difficult to ascertain which of these our task may precisely relate to (Shettleworth, 1993). It also remains possible that multiple long-term memory systems underlie performance on these visual discrimination tasks (Roberts et al., 2015). One type of long-term visual memory that has previously been assessed in another species of Galliform is declarative memory (Forkman, 2000). Declarative memory involves "knowing that" in contrast to non-declarative, procedural or kinesthetic memory, which are terms often used to describe "knowing how" i.e., the learning of motor skills (Roediger et al., 2009). However, declarative memory is also a general definition that includes both semantic memory (general knowledge) and episodic memory (remembering specific events of the past). Hence it is likely that the long-term reference memory assessed in the current study also involves declarative and episodic processes. Although domestic hens have shown capacities to form declarative representations of color associations (Forkman, 2000), little is known about long-term memory retention in other Galliformes (Marino, 2017). However, we may expect such capacities, as the ability to learn and recall multiple discriminations is seen in other birds, such as white Carneaux pigeons (Columbia livia), which can learn 160 pairs of different pictures and recall these items over periods of up to 731 days (Vaughan \& Greene, 1984), and Silver King pigeons which show an accuracy of $73 \%$ on a memory set of over 1,800 pictures (Cook et al., 2005). Interestingly, pheasants recalled the rewarded color of a pair, even after subsequently experiencing new associations of a further four color pairs. In addition to the lack of temporal decay of learned associations, young pheasants also recalled both initially learnt and subsequently learnt discriminations equally well. In the wild, such capacities may facilitate responses to the seasonal availability of different food types associated with their generalist diet, or help when recalling the visual identity of individuals to maintain social affiliations across breeding seasons as required by their fission-fusion social dynamics (Hill \& Robertson, 1988). Indeed, the ability to recall seasonally available foods has been considered important for maintaining long-term memories in other species (Soldati, Burman, John, Pike, \& Wilkinson, 2017). Likewise, long-term memories of social relationships are often found among species that congregate seasonally (Godard, 1991; Insley, 2000), or live in complex fission-fusion social groups (Boeckle \& Bugnyar, 2012). However, as long-term memory encompasses many kinds of memory and can also involve multiple memory systems, it is likely that a number of different processes are responsible for learning and recalling different stimuli. For example, remembering the visual identity of someone may involve different kinds of memory than those required to remember different colors. Future studies may therefore benefit by investigating different forms of memory in Galliformes, for example by assessing recall of pattern discrimination problems or procedural tasks, which may subsequently highlight whether differences in memory on specific tasks are related to general or domain specific cognitive processes. Similar approaches have illuminated adaptive specializations for spatial memory in caching birds (Clayton \& Krebs, 1994). Future studies could also counterbalance the presentation order of successive color pairs during the discrimination learning phase to investigate whether pheasants, or other Galliformes, show improvements through processes of generalized rule learning, as has been found in other species of birds (Hunter \& Kamil, 1971; van Horik \& Emery, 2016; Wilson, Mackintosh, \& Boakes, 1985).

We found no evidence that individual performances were repeatable, within learning and memory tasks, or across learning and memory tasks. Mixed findings have been reported on the consistency of performance on discrimination tasks in guinea pigs, Cavia aperea f. porcellus, (Brust \& Guenther, 2017; Guenther \& Brust, 2017). However, such stability in cognitive traits is rarely reported in animals (Griffin, Guillette, \& Healy, 2015; Sih \& Del Giudice, 2012). However, pheasants that made more overall correct choices on the learning discriminations also made more overall correct choices on the memory 
discriminations. This may be because rapid learners experienced a stronger reinforcement history of each association, which may facilitate their ability to recall each color pair. Similar findings have, however, been reported in bumblebees using similar color discrimination paradigms, with faster learners showing greater overnight retention of previously learned information as well as showing greater flexibility in their ability to respond to reversed contingencies of previously learned colors (Raine \& Chittka, 2012). Subsequent studies could investigate the relationship between learning reinforcement history and memory further by training subjects to a predetermined learning criterion before re-testing them at varying intervals in time.

In conclusion, we found that young pheasants were capable of successively learning the reward contingencies of five different color pairs and could retain these associations over intervals of at least 27 days. These findings suggest pheasants are capable of forming long-term reference memories of a declarative nature. We also found that an individual's learning performance correlated positively with their memory performance. Our findings therefore illuminate previously unreported cognitive capacities in a species of Galliforme, the pheasant.

\section{Conflict of interest}

The authors declare no conflict of interest.

\section{Acknowledgements}

JRM, MAW and JOvH were funded by an ERC consolidator grant (616474). Rothamsted Research, North Wyke hosted the rearing and release of the pheasants. Kenzie Bess, Molly Watts and Heather Warrender helped with data collection and animal husbandry. Pip Laker provided comments on the manuscript.

\section{References}

Balda, R. P., \& Kamil, A. C. (1992). Long-term spatial memory in Clark nutcracker, Nucifraga columbiana. Animal Behaviour, 44, 761-769.

Bates, D., Maechler, M., Bolker, B., \& Walker, S. (2015). Fitting linear mixed-effects models using lme4. Journal of Statistical Software, 67, 1-48.

Bird, C. D., \& Emery, N. J. (2008). Using video playback to investigate the social preferences of rooks, Corvus frugilegus. Animal Behaviour, 76, 679-687.

Boeckle, M., \& Bugnyar, T. (2012). Long-term memory for affiliates in ravens. Current Biology, 22, 801-806.

Boyer, D., \& Walsh, P. D. (2010). Modelling the mobility of living organisms in heterogeneous landscapes: Does memory improve foraging success? Philosophical Transactions. Series A, Mathematical, Physical, and Engineering Sciences, 368, 5645-5659.

Bradshaw, R. H. (1991). Discrimination of group members by laying hens, Gallus domesticus. Behavioural Processes, 24, 143-151.

Brown, G. D. A., Neath, I., \& Chater, N. (2007). A temporal ratio model of memory. Psychological Review, 114, 539-576.

Brust, V., \& Guenther, A. (2017). Stability of the guinea pigs personality - cognition - linkage over time. Behavioural Processes, 134, 4-11.

Churchland, P. S. (1986). Neurophilosophy: Toward a unified science of the mind-brain. Cambridge, MA: MIT Press.

Clayton, N. S., \& Dickinson, A. (1998). Episodic-like memory during cache recovery by scrubjays. Nature, 395, $272-274$.

Clayton, N. S., \& Krebs, J. R. (1994). Memory for spatial and object-specific cues in food-storing and non-storing birds. Journal of Comparative Physiology A, 174, 371-379.

Cook, R. G., Levison, D. G., Gillett, S. R., \& Blaisdell, A. P. (2005). Capacity and limits of associative memory in pigeons. Psychonomic Bulletin and Review, 12, 350-358.

DEFRA. (2009). Code of practice for welfare of game birds reared for sporting purposes. 
Dudchenko, P. A. (2004). An overview of the tasks used to test working memory in rodents. Neuroscience and Biobehavioral Reviews, 28, 699-709.

Forkman, B. (2000). Domestic hens have declarative representations. Animal Cognition, 3, 135-137.

Gaffan, E. A. (1992). Primacy, recency, and the variability of data in studies of animals' working memory. Animal Learning \& Behavior, 20, 240-252.

Godard, R. (1991). Long-term memory of individual neighbours in a migratory songbird. Nature, 350, 228-229.

Griffin, A. S., Guillette, L. M., \& Healy, S. D. (2015). Cognition and personality: An analysis of an emerging field. Trends in Ecology and Evolution, 30, 207-214.

Guenther, A., \& Brust, V. (2017). Individual consistency in multiple cognitive performance: Behavioural versus cognitive syndromes. Animal Behaviour, 130, 119-131.

Healy, S. D., \& Jones, C. M. (2002). Animal learning and memory: An integration of cognition and ecology. Zoology (Jena, Germany), 105, 321-327.

Hill, D., \& Robertson, P. (1988). The pheasant: Ecology, management and conservation. London: BSP Professional Books, Blackwell Scientific Publications Ltd.

Honig, W. (1978). Studies of working memory in the pigeon. In S. Hulse, H. Fowler, \& W. Honig (Eds.), Cognitive processes in animal behaviour (p. 211-248). Hillsdale, NJ: Erlbaum.

Honig, W. K., \& James, P. H. R. (1971). Animal Memory. New York: Academic Press.

Hunter, M. W., \& Kamil, A. C. (1971). Object-discrimination learning set and hypothesis behavior in the northern bluejay (Cynaocitta cristata). Psychonomic Science, 22, 271-273.

IBM Corp. (2013). IBM SPSS statistics for windows, Version 22.0. New York: Armonk.

Insley, S. J. (2000). Long-term vocal recognition in the northern fur seal. Nature, 406, 404-405.

Keppel, G., \& Underwood, B. J. (1962). Proactive inhibition in short-term retention of single items. Journal of Verbal Learning and Verbal Behavior, 1, 153-161.

Lohmann, K. J., Luschi, P., \& Hays, G. C. (2008). Goal navigation and island-finding in sea turtles. Journal of Experimental Marine Biology and Ecology, 356, 83-95.

Mackintosh, N. J. (1974). The psychology of animal learning. New York: Academic Press.

Marino, L. (2017). Thinking chickens: A review of cognition, emotion, and behavior in the domestic chicken. Animal Cognition, 20, 127-147.

Mery, F., \& Kawecki, T. J. (2005). A cost of long-term memory in drosophila. Science, 308, 1148-1148.

Mettke-Hofmann, C., \& Gwinner, E. (2003). Long-term memory for a life on the move. Proceedings of the National Academy of Sciences of the United States of America, 100, 5863-5866.

Mulcahy, N. J., \& Call, J. (2006). Apes save tools for future use. Science, 312, 1038-1040.

Nakagawa, S., \& Schielzeth, H. (2010). Repeatability for Gaussian and non-Gaussian data: A practical guide for biologists. Biological Reviews, 85, 935-956.

Okano, H., Hirano, T., \& Balaban, E. (2000). Learning and memory. Proceedings of the National Academy of Sciences of the United States of America, 97, 12403-12404.

Olton, D. S., Collison, C., \& Werz, M. A. (1977). Spatial memory and radial arm maze performance of rats. Learning and Motivation, 8, 289-314.

Osvath, M. (2009). Spontaneous planning for future stone throwing by a male chimpanzee. Current Biology, 19, 190-191.

Raby, C. R., Alexis, D. M., Dickinson, A., \& Clayton, N. S. (2007). Planning for the future by western scrub-jays. Nature, 445, 919-21. https://doi.org/10.1038/nature05575

Raby, C. R., \& Clayton, N. S. (2009). Prospective cognition in animals. Behavioural Processes, 80, 314-324. https://doi.org/10.1016/j.beproc.2008.12.005

$\mathrm{R}$ Development Core Team. (2014). R: A language and environment for stastical computing. Vienna, Austria: $\mathrm{R}$ Foundation for Statistical Computing.

Raine, N. E., \& Chittka, L. (2008). The correlation of learning speed and natural foraging success in bumble-bees. Proceedings of the Royal Society B: Biological Sciences, 275, 803-808.

Raine, N. E., \& Chittka, L. (2012). No trade-off between learning speed and associative flexibility in bumblebees: A reversal learning test with multiple colonies. PLOS ONE, 7, e45096.

Rescorla, R. A. (1988). Pavlovian conditioning. It's not what you think it is. American Psychologist, 43, 151-160.

Roberts, W. A., Strang, C., \& Macpherson, K. (2015). Memory systems interaction in the pigeon: Working and reference memory. Journal of Experimental Psychology: Animal Learning and Cognition, 41, 152-162.

Roediger, H. L., Zaromb, F. M., \& Goode, M. K. (2009). A typology of memory terms. In J. H. Byrne (Ed.), Concise learning and memory: The editor's selection (p. 1-15). London: Academic Press. 
Roth, T. C., LaDage, L. D., Freas, C. A., \& Pravosudov, V. V. (2012). Variation in memory and the hippocampus across populations from different climates: A common garden approach. Proceedings of the Royal Society B: Biological Sciences, 279, 402-410.

Roth, T. C., \& Pravosudov, V. V. (2009). Hippocampal volumes and neuron numbers increase along a gradient of environmental harshness: A large-scale comparison. Proceedings. Biological Sciences / The Royal Society, 276, 401-405.

Sands, S., \& Wright, A. (1980). Primate memory: Retention of serial list items by a rhesus monkey. Science, 209, 938-940.

Santiago, H. C., \& Wright, A. A. (1984). Pigeon memory: Same/different concept learning, serial probe recognition acquisition, and probe delay effects on the serial-position function. Journal of Experimental Psychology: Animal Behavior Processes, 10, 498-512.

Shettleworth, S. J. (1993). Varieties of learning and memory in animals. Journal of Experimental Psychology. Animal Behavior Processes, 19, 5-14.

Shettleworth, S. J. (2010). Cognition, evolution, and behavior, 2nd ed. New York: Oxford University Press.

Sih, A., \& Del Giudice, M. (2012). Linking behavioural syndromes and cognition: A behavioural ecology perspective. Philosophical Transactions of the Royal Society B-Biological Sciences, 367, 2762-2772.

Soldati, F., Burman, O. H. P., John, E. A., Pike, T. W., \& Wilkinson, A. (2017). Long-term memory of relative reward values. Biology Letters, 13: 0160853.

Stoffel, M. A., Nakagawa, S., \& Schielzeth, H. (2017). rptR: Repeatability estimation and variance decomposition by generalized linear mixed-effects models. Methods in Ecology and Evolution, 8, 1639-1644.

Tulving, E. (2007) Are There 256 Different Kinds of Memory? in J.S. Nairne (ed.) The Foundations of Remembering: Essays in Honor of Henry L. Roediger, III, pp. 39-52. New York: Psychology Press.

van Horik, J., \& Burns, K. C. (2007). Cache spacing patterns and reciprocal cache theft in New Zealand robins. Animal Behaviour, 73, 1043-1049.

van Horik, J. O., \& Emery, N. J. (2016). Transfer of physical understanding in a non-tool-using parrot. Animal Cognition, 19, 1195-1203.

van Horik, J. O., Langley, E. J. G., Whiteside, M. A., \& Madden, J. R. (2017). Differential participation in cognitive tests is driven by personality, sex, body condition and experience. Behavioural Processes, 134, 22-30.

Vaughan, W., \& Greene, S. L. (1984). Pigeon visual memory capacity. Journal of Experimental Psychology: Animal Behavior Processes, 10, 256-271.

Wascher, C. A. F., Szipl, G., Boeckle, M., \& Wilkinson, A. (2012). You sound familiar: Carrion crows can differentiate between the calls of known and unknown heterospecifics. Animal Cognition, 15, 1015-1019.

Wilson, B., Mackintosh, N. J., \& Boakes, R. A. (1985). Transfer of relational rules in matching and oddity learning by pigeons and corvids. The Quarterly Journal of Experimental Psychology Section B, 37(789998259), 313332.

Wright, A., Santiago, H., Sands, S., Kendrick, D., \& Cook, R. (1985). Memory processing of serial lists by pigeons, monkeys, and people. Science, 229, 287-289. 